\title{
THE HASSE NORM PRINCIPLE FOR $A_{n}$-EXTENSIONS
}

\author{
ANDRÉ MACEDO
}

\begin{abstract}
We prove that, for every $n \geq 5$, the Hasse norm principle holds for a degree $n$ extension $K / k$ of number fields with normal closure $F$ such that $\operatorname{Gal}(F / k) \cong A_{n}$. We also show the validity of weak approximation for the associated norm one tori.
\end{abstract}

\section{INTRODUCTION}

Let $K / k$ be an extension of number fields with associated idèle groups $\mathbb{A}_{K}^{*}$ and $\mathbb{A}_{k}^{*}$ and let $\mathrm{N}_{K / k}: \mathbb{A}_{K}^{*} \rightarrow \mathbb{A}_{k}^{*}$ be the norm map on the idèles. We can view $K^{*}$ (respectively, $k^{*}$ ) as sitting inside $\mathbb{A}_{K}^{*}$ (respectively, $\mathbb{A}_{k}^{*}$ ) via the diagonal embedding and $\mathrm{N}_{K / k}$ naturally extends the usual norm map of the extension $K / k$. We say that the Hasse norm principle (often abbreviated to HNP) holds for $K / k$ if the knot group

$$
\mathfrak{K}(K / k):=\left(k^{*} \cap \mathrm{N}_{K / k}\left(\mathbb{A}_{K}^{*}\right)\right) / \mathrm{N}_{K / k}\left(K^{*}\right)
$$

is trivial, i.e. if every nonzero element of $k$ which is a local norm everywhere is a global norm.

The first example of the validity of this principle was established in 1931 by Hasse, who proved that the knot group $\mathfrak{K}(K / k)$ is trivial if $K / k$ is a cyclic extension (the Hasse norm theorem). Since then, much work has been done in the abelian case (see, for instance, [7], [9] or [12]), but results for the non-abelian and non-Galois cases are still limited. For example, if $F$ denotes the normal closure of $K / k$, it is known that the HNP holds for $K / k$ when

- $[K: k]$ is prime $([1])$;

- $[K: k]=n$ and $\operatorname{Gal}(F / k) \cong D_{n}$, the dihedral group of order $2 n([2])$;

- $[K: k]=n$ and $\operatorname{Gal}(F / k) \cong S_{n}$, the symmetric group on $n$ letters ([20]).

In this paper, we study the HNP for a degree $n$ extension $K / k$ with normal closure $F$ such that $\operatorname{Gal}(F / k)$ is isomorphic to $A_{n}$, the alternating group on $n$ letters. We also look at weak approximation - recall that this property is said to hold for a variety $X / k$ if $X(k)$ is dense (for the product topology) in $\prod_{v} X\left(k_{v}\right)$, where the product is taken over all places $v$ of $k$ and $k_{v}$ denotes the completion of $k$ with respect to $v$. In particular, we examine weak approximation for the norm one torus $T=R_{K / k}^{1} \mathbb{G}_{m}$ associated to a degree $n$ extension of number fields $K / k$ with $A_{n}$-normal closure.

The first non-trivial case is $n=3$. In this case, $K=F$ is a Galois extension of $k$ and the Hasse norm theorem tells us that the HNP holds for $K / k$. Moreover, using a result of Voskresenskiŭ, one can show that weak approximation holds for

2010 Mathematics Subject Classification. 14G05 (primary), 11E72, 11R37, 20D06 (secondary). 
the associated norm one torus. In [15] Kunyavskiur solved the case $n=4$ by showing that, for a quartic extension $K / k$ with $A_{4}$-normal closure, $\mathfrak{K}(K / k)$ is either 0 or $\mathbb{Z} / 2$ and both cases can occur. Additionally, he proved that the HNP holds for $K / k$ if and only if weak approximation fails for $T$. In this paper, we use several cohomological results about $A_{n}$-modules to prove the following theorem.

Theorem 1.1. Let $n \geq 5$ be an integer. Let $K / k$ be a degree $n$ extension of number fields and let $F$ be its normal closure. If $\mathrm{Gal}(F / k) \cong A_{n}$, then the Hasse norm principle holds for $K / k$ and weak approximation holds for the norm one torus $T=R_{K / k}^{1} \mathbb{G}_{m}$.

The layout of this paper is as follows. In Section 2, we use various cohomological and group-theoretic tools to establish the injectivity of an important restriction map on the cohomology of $A_{n}$. In Section 3, we look at the consequences of these results in the arithmetic of number fields. In particular, combining the results of Section 2 with the work of Colliot-Thélène and Sansuc on flasque resolutions and a theorem of Voskresenskiú, we prove Theorem 1.1 for $n \neq 6$. In Section 4, we exploit a computational method developed by Hoshi and Yamasaki to solve the remaining case $n=6$. All of the code used in Section 4 is provided in the Appendix.

Notation. Throughout this paper, we fix the following notation.

$k \quad$ a number field;

$\bar{k} \quad$ an algebraic closure of $k$;

$v \quad$ a place of $k$;

$k_{v} \quad$ the completion of $k$ at $v$.

For a variety $X$ over a field $K$, we use the notation

$X_{L}=X \times_{K} L \quad$ the base change of $X$ to a field extension $L / K$;

$\bar{X}=X \times_{K} \bar{K} \quad$ the base change of $X$ to an algebraic closure of $K$;

Pic $X \quad$ the Picard group of $X$.

We define $\mathbb{G}_{m, K}=\operatorname{Spec}\left(K\left[t, t^{-1}\right]\right)$ to be the multiplicative group over a field $K$ and, if $K$ is apparent from the context, we omit it from the subscript and simply write $\mathbb{G}_{m}$. Given a $K$-torus $T$, we write $\hat{T}$ for its character group $\operatorname{Hom}\left(\bar{T}, \mathbb{G}_{m, \bar{K}}\right)$. If $L / K$ is a finite extension of fields and $T$ is an $L$-torus, we denote the Weil restriction of $T$ from $L$ to $K$ by $R_{L / K} T$. We use the notation $R_{L / K}^{1} \mathbb{G}_{m}$ for the norm one torus, defined as the kernel of the norm map $\mathrm{N}_{L / K}: R_{L / K} \mathbb{G}_{m} \rightarrow \mathbb{G}_{m}$.

Let $G$ be a finite group. The label ' $G$-module' shall always mean a free $\mathbb{Z}$ module of finite rank equipped with a right action of $G$. For a $G$-module $A$ and $q \in \mathbb{Z}$, we denote the Tate cohomology groups by $\hat{\mathrm{H}}^{q}(G, A)$ and the kernel of the restriction map $\hat{\mathrm{H}}^{q}(G, A) \stackrel{\text { Res }}{\longrightarrow} \prod_{g \in G} \hat{\mathrm{H}}^{q}(\langle g\rangle, A)$ by $\amalg_{\omega}^{q}(G, A)$. Since $\hat{\mathrm{H}}^{q}(G, A)=$ $\mathrm{H}^{q}(G, A)$ for $q \geq 1$, we will omit the hat in this case. We also use the notation $Z(G),[G, G], G^{\sim}$ and $M(G)$ for the center, the derived subgroup, the dual group $\operatorname{Hom}(G, \mathbb{Q} / \mathbb{Z})$ and the Schur multiplier $\hat{\mathrm{H}}^{-3}(G, \mathbb{Z})$ of $G$, respectively. Given elements $g, h \in G$, we use the conventions $[g, h]=g h g^{-1} h^{-1}$ and $g^{h}=h g h^{-1}$. 
Acknowledgements. I would like to thank my supervisor Rachel Newton for suggesting this problem and for helpful comments on an earlier version of this manuscript. I am also grateful to Prof. Boris Kunyavskiu for bringing up the importance of Lemma 2.2. This work was supported by the FCT doctoral scholarship SFRH/BD/117955/2016.

\section{Group Cohomology of $A_{n}$-Modules}

The goal of this section is to establish several cohomological facts about $A_{n^{-}}$ modules. We start by stating some useful group-theoretic facts.

Remark 2.1. Recall that, for $n \geq 5, A_{n}$ is a non-abelian simple group and hence perfect. Moreover, its Schur multiplier $M\left(A_{n}\right)=\hat{\mathrm{H}}^{-3}\left(A_{n}, \mathbb{Z}\right)$ is given as follows (see Theorem 2.11 of [11]):

$$
M\left(A_{n}\right)= \begin{cases}0 & \text { if } n \leq 3 \\ \mathbb{Z} / 2 & \text { if } n \in\{4,5\} \text { or } n \geq 8 \\ \mathbb{Z} / 6 & \text { if } n \in\{6,7\} .\end{cases}
$$

Given a copy $H$ of $A_{n-1}$ inside $G=A_{n}$, we have a corestriction map on cohomology

$$
\operatorname{Cor}_{G}^{H}: M(H) \rightarrow M(G)
$$

This map will play an important role for us later, so we begin by establishing the following result.

Lemma 2.2. Let $n \geq 8$ and let $H$ be a copy of $A_{n-1}$ inside $G=A_{n}$. Then, the corestriction map $\mathrm{Cor}_{G}^{H}$ is surjective.

In order to prove this lemma, we will use multiple results about covering groups of $S_{n}$ and $A_{n}$ together with the characterization of the image of $\operatorname{Cor}_{G}^{H}$ given in Lemma 4 of [6]. To put this plan into practice, we need the following concepts.

Definition 2.3. Let $G$ be a finite group. A stem extension of $G$ is a group $\widetilde{G}$ containing a normal subgroup $K$ such that $\widetilde{G} / K \cong G$ and $K \subseteq Z(\widetilde{G}) \cap[\widetilde{G}, \widetilde{G}]$. A Schur covering group of $G$ is a stem extension of $G$ of maximal size.

It is a well-known fact that a stem extension of a finite group $G$ always exists (see Theorem 2.1.4 of [14]). Additionally, the base normal subgroup $K$ of a Schur covering group $\widetilde{G}$ of $G$ coincides with its Schur multiplier $\hat{\mathrm{H}}^{-3}(G, \mathbb{Z})$ (see Section 9.9 of [10]). In [18], Schur completely classified the Schur covering groups of $S_{n}$ and $A_{n}$. He also gave an explicit presentation of a cover of $S_{n}$, as follows.

Proposition 2.4. Let $n \geq 4$ and let $U$ be the group with generators $z, t_{1}, \cdots, t_{n-1}$ and relations

(1) $z^{2}=1$

(2) $z t_{i}=t_{i} z$, for $1 \leq i \leq n-1$;

(3) $t_{i}^{2}=z$, for $1 \leq i \leq n-1$;

(4) $\left(t_{i} t_{i+1}\right)^{3}=z$, for $1 \leq i \leq n-2$;

(5) $t_{i} t_{j}=z t_{j} t_{i}$, for $|i-j| \geq 2$ and $1 \leq i, j \leq n-1$. 
Then $U$ is a Schur covering group of $S_{n}$ with base normal subgroup $K=\langle z\rangle$. Moreover, if $\overline{t_{i}}$ denotes the transposition $(i i+1)$ in $S_{n}$, then the map

$$
\begin{aligned}
\pi: U & \longrightarrow S_{n} \\
z & \longmapsto 1 \\
t_{i} & \longmapsto \overline{t_{i}}
\end{aligned}
$$

is surjective and has kernel $K$.

Proof. See Schur's original paper [18] or Chapter 2 of [11] for a more modern exposition.

Remark 2.5. An immediate consequence of this last proposition is that the Schur multiplier of $S_{n}$ is isomorphic to $\mathbb{Z} / 2$ for $n \geq 4$.

Using the Schur cover of $S_{n}$ given in Proposition 2.4, one can also construct a Schur covering group of $A_{n}$ for $n \geq 8$.

Lemma 2.6. In the notation of Proposition 2.4, the group $V:=\pi^{-1}\left(A_{n}\right)$ defines a Schur covering group of $A_{n}$ for every $n \geq 8$.

Proof. It is well-known that $A_{n}$ is generated by the $n-2$ permutations $\overline{e_{i}}:=$ $\overline{t_{1}} \cdot \overline{t_{i+1}}=\left(\begin{array}{ll}1 & 2\end{array}\right)(i+1 i+2)$ for $1 \leq i \leq n-2$. Hence, $V=\pi^{-1}\left(A_{n}\right)$ is generated by $z, e_{1}, \cdots, e_{n-2}$, where $e_{i}:=t_{1} t_{i+1}$ for $1 \leq i \leq n-2$. Clearly, we have $K \subseteq Z(V)$ and $V / K \cong A_{n}$. As the Schur multiplier of $A_{n}$ is also $\mathbb{Z} / 2$ for $n \geq 8$, in order to show that $V$ defines a Schur covering group of $A_{n}$ it suffices to prove that $K \subseteq[V, V]$.

Claim: $z=\left[e_{1}^{-1} e_{2} e_{1}, e_{2}\right]$.

Proof of claim: This follows from a standard computation using the identities $\left(e_{1} e_{2}\right)^{3}=z, e_{1}^{3}=z$ and $e_{i}^{2}=z$ for $2 \leq i \leq n-2$, which follow directly from the relations satisfied by the $t_{i}$.

Given the claim, it follows that $K=\langle z\rangle$ is contained in $[V, V]$, as desired.

Given a copy $H$ of $A_{n-1}$ inside $A_{n}$, one can subsequently repeat the same procedure of this last lemma and further restrict $\pi$ to $W:=\pi^{-1}(H)$ to seek a Schur covering group of $H$. The same argument works, but with two small caveats.

First, it is necessary to assure that we still have $z \in[W, W]$. This is indeed the case since, for $n \geq 7$, any subgroup $H \leq A_{n}$ isomorphic to $A_{n-1}$ is conjugate to the point stabilizer $\left(A_{n}\right)_{n}$ of the letter $n$ in $A_{n}$ (this is a consequence of Lemma 2.2 of [21]). Therefore, we have $H=\left(A_{n}\right)_{n}{ }^{\pi(x)}$ for some $x \in U$ and hence $z=z^{x}=\left[e_{1}^{-1} e_{2} e_{1}, e_{2}\right]^{x}=\left[\left(e_{1}^{-1} e_{2} e_{1}\right)^{x}, e_{2}^{x}\right]$ is in $[W, W]$, as clearly $\overline{e_{1}}, \overline{e_{2}} \in\left(A_{n}\right)_{n}$.

Second, note that we are making use of the fact that the Schur multipliers of $A_{n-1}$ and $S_{n}$ coincide, which is only true for $n \geq 9$ (recall that $M\left(A_{7}\right)=\mathbb{Z} / 6$ ). However, it is still true that $\pi^{-1}\left(A_{7}\right)$ gives a (non-maximal) stem extension of $A_{7}$ by the same reasoning as above. We have thus established the following result. 
Lemma 2.7. Let $n \geq 8$ and let $H$ be a copy of $A_{n-1}$ inside $A_{n}$. Then, the restriction to $W=\pi^{-1}(H)$ of the Schur cover $V$ of $A_{n}$ given in Lemma 2.6 defines a stem extension of $H$.

We can now prove Lemma 2.2.

Proof of Lemma 2.2. Let $V$ be the Schur covering group of $G$ constructed in Lemma 2.6. We then have a central extension

$$
1 \rightarrow M(G) \rightarrow V \stackrel{\pi}{\rightarrow} G \rightarrow 1
$$

where we identified the base normal subgroup $K$ of $V$ with the Schur multiplier $M(G)$ of $G$. Since $M(G) \subset[V, V]$ by the definition of a Schur cover, $V$ is a generalized representation group of $G$, as defined on p. 310 of [6]. Therefore, by Lemma 4 of [6], we have an isomorphism $\operatorname{Cor}_{G}^{H}(M(H)) \cong M(G) \cap[W, W]$, where $W=\pi^{-1}(H)$. Hence, it is enough to show that $M(G) \cap[W, W]=M(G)$. By Lemma 2.7. $W$ defines a stem extension of $H$ for $n \geq 8$, so that we immediately get $M(G) \subset[W, W]$. It follows that $M(G) \cap[W, W]=M(G)$, as desired.

In order to proceed with our cohomological analysis, we need to recollect some group-theoretic objects. Let $H$ be a subgroup of a finite group $G$. Recall that we have the augmentation map $\epsilon: \mathbb{Z}[G / H] \rightarrow \mathbb{Z}$ defined by $\epsilon: H g \mapsto 1$ for any $H g \in G / H$. This map produces the exact sequence of $G$-modules

$$
0 \rightarrow I_{G / H} \rightarrow \mathbb{Z}[G / H] \stackrel{\epsilon}{\rightarrow} \mathbb{Z} \rightarrow 0
$$

where $I_{G / H}=\operatorname{ker}(\epsilon)$ is the augmentation ideal. Dually, we also have a map $\eta: \mathbb{Z} \rightarrow \mathbb{Z}[G / H]$ defined by $\eta: 1 \mapsto N_{G / H}$, where $N_{G / H}=\sum_{H \in G / H} H g$. This produces the exact sequence of $G$-modules

$$
0 \rightarrow \mathbb{Z} \stackrel{\eta}{\rightarrow} \mathbb{Z}[G / H] \rightarrow J_{G / H} \rightarrow 0
$$

where $J_{G / H}=\operatorname{coker}(\eta)$ (called the Chevalley module of $G / H$ ) is the dual module $\operatorname{Hom}\left(I_{G / H}, \mathbb{Z}\right)$ of $I_{G / H}$.

For any $g \in G$, we can consider the restriction maps

$$
\operatorname{Res}_{g}: \mathrm{H}^{2}\left(G, J_{G / H}\right) \rightarrow \mathrm{H}^{2}\left(\langle g\rangle, J_{G / H}\right)
$$

and aggregate all of these functions together in order to get a homomorphism of $G$-modules

$$
\text { Res : } \mathrm{H}^{2}\left(G, J_{G / H}\right) \rightarrow \prod_{g \in G} \mathrm{H}^{2}\left(\langle g\rangle, J_{G / H}\right) .
$$

It turns out that the kernel of this map (denoted by $\amalg_{\omega}^{2}\left(G, J_{G / H}\right)$ ) is of extreme importance in the arithmetic of number fields, as we will see in the next section. We describe this kernel for our case of interest $G=A_{n}, H \cong A_{n-1}$ and $n \geq 8$ (the cases $n \leq 7$ will be treated separately). 
Proposition 2.8. Let $n \geq 8$ and let $H$ be a copy of $A_{n-1}$ inside $G=A_{n}$. Then, we have $\amalg_{\omega}^{2}\left(G, J_{G / H}\right)=0$.

Proof. Taking the $G$-cohomology of the exact sequence $(2.2)$ gives the exact sequence of abelian groups

$$
\mathrm{H}^{2}(G, \mathbb{Z}[G / H]) \rightarrow \mathrm{H}^{2}\left(G, J_{G / H}\right) \rightarrow \mathrm{H}^{3}(G, \mathbb{Z}) \stackrel{\bar{\eta}}{\rightarrow} \mathrm{H}^{3}(G, \mathbb{Z}[G / H]),
$$

where $\bar{\eta}$ is the map induced on the degree 3 cohomology groups by the norm map $\eta$. Applying Shapiro's lemma and using the fundamental duality theorem in the cohomology of finite groups (see, for example, Section VI.7 of [3]), we have $\mathrm{H}^{2}(G, \mathbb{Z}[G / H]) \cong \mathrm{H}^{2}(H, \mathbb{Z}) \cong \hat{\mathrm{H}}^{-2}(H, \mathbb{Z}) \cong H /[H, H]=0$, as $H$ is perfect. Therefore, this last exact sequence becomes

$$
0 \rightarrow \mathrm{H}^{2}\left(G, J_{G / H}\right) \rightarrow \mathrm{H}^{3}(G, \mathbb{Z}) \stackrel{\bar{\eta}}{\rightarrow} \mathrm{H}^{3}(G, \mathbb{Z}[G / H]),
$$

which shows that $\mathrm{H}^{2}\left(G, J_{G / H}\right)=0$ if $\bar{\eta}$ is injective. Since the composition of the map $\bar{\eta}$ with the isomorphism in Shapiro's lemma

$$
\mathrm{H}^{3}(G, \mathbb{Z}) \stackrel{\bar{\eta}}{\rightarrow} \mathrm{H}^{3}(G, \mathbb{Z}[G / H]) \stackrel{\cong}{\rightarrow} \mathrm{H}^{3}(H, \mathbb{Z})
$$

gives the restriction map (see Example 1.27(b) of [16]), it is enough to prove that the restriction

$$
\operatorname{Res}_{H}^{G}: \mathrm{H}^{3}(G, \mathbb{Z}) \rightarrow \mathrm{H}^{3}(H, \mathbb{Z})
$$

is injective. Again, by the duality in the cohomology of finite groups, this is the same as proving that the corestriction map (dual to $\operatorname{Res}_{H}^{G}$ )

$$
\operatorname{Cor}_{G}^{H}: \hat{\mathrm{H}}^{-3}(H, \mathbb{Z}) \rightarrow \hat{\mathrm{H}}^{-3}(G, \mathbb{Z})
$$

is surjective. But this is the content of Lemma 2.2 , so it follows that $\mathrm{H}^{2}\left(G, J_{G / H}\right)$ is trivial and therefore $\amalg_{\omega}^{2}\left(G, J_{G / H}\right)=0$, as desired.

\section{Arithmetic COnsequences}

In this section, we delve into the consequences of the cohomological results of Section 2 in the arithmetic of number fields. In particular, we will recall how the group $\amalg_{\omega}^{2}\left(G, J_{G / H}\right)$ governs two important local-global principles, the Hasse norm principle and weak approximation. Specifying to the case $G=A_{n}$, $H \cong A_{n-1}$ and using Proposition 2.8, we will prove Theorem 1.1 for $n \geq 8$. The remaining cases $(n \leq 7)$ will be solved using a result of Colliot-Thélène and Sansuc and a computational method adapted from work of Hoshi and Yamasaki.

Let $k$ be a number field and let $T$ be a $k$-torus. We introduce the defect to weak approximation for $T$

$$
A(T)=\left(\prod_{v} T\left(k_{v}\right)\right) / \overline{T(k)}
$$


where the product is taken over all places $v$ of $k$ and $\overline{T(k)}$ denotes the closure (with respect to the product topology) of $T(k)$ in $\prod_{v} T\left(k_{v}\right)$. We say that weak approximation holds for $T$ if and only if $A(T)=0$.

We also define the Tate-Shafarevich group of $T$ as

$$
\amalg(T)=\operatorname{ker}\left(\mathrm{H}^{1}(k, T) \rightarrow \prod_{v} \mathrm{H}^{1}\left(k_{v}, T_{k_{v}}\right)\right),
$$

where the product runs over all places $v$ of $k$. It is known that this group controls the validity of the Hasse principle for every principal homogeneous space under $T$. In fact, the Hasse principle holds for every such space if and only if $\amalg(T)=0$.

The following result remarkably connects weak approximation with the Hasse principle by combining the two groups $A(T)$ and $\amalg(T)$ in an exact sequence.

Theorem 3.1 (Voskresenskiū). Let $T$ be a torus defined over a number field $k$ and let $X / k$ be a smooth projective model of $T$. Then there exists an exact sequence

$$
0 \rightarrow A(T) \rightarrow \mathrm{H}^{1}(k, \operatorname{Pic} \bar{X})^{\sim} \rightarrow \amalg(T) \rightarrow 0 .
$$

Proof. See Theorem 6 of [19].

Let us now specialize $T$ to be the norm one torus $R_{K / k}^{1} \mathbb{G}_{m}$ of an extension $K / k$ of number fields. In this case, we have $\mathfrak{K}(K / k) \cong \amalg(T)$ (see p. 307 of [17]). Therefore, the cohomology group $\mathrm{H}^{1}(k, \operatorname{Pic} \bar{X})$ in the previous theorem is pivotal in the study of the HNP for $K / k$ and weak approximation for $T$. A very useful tool to deal with this object is flasque resolutions, as introduced in the work of Colliot-Thélène and Sansuc. We recall here the main definitions and refer the reader to [4] and [5] for more details on this topic.

Flasque resolutions. Let $G$ be a finite group and let $A$ be a $G$-module. The module $A$ is said to be flasque if $\hat{\mathrm{H}}^{-1}\left(G^{\prime}, A\right)=0$ for every subgroup $G^{\prime}$ of $G$ and coflasque if $\mathrm{H}^{1}\left(G^{\prime}, A\right)=0$ for every subgroup $G^{\prime}$ of $G$. Moreover, $A$ is called a permutation module if it admits a $\mathbb{Z}$-basis permuted by $G$ and an invertible module if it is a direct summand of a permutation module. A flasque resolution of $A$ is an exact sequence of $G$-modules

$$
0 \rightarrow A \rightarrow P \rightarrow M \rightarrow 0
$$

where $P$ is a permutation module and $M$ is flasque. Dually, a coflasque resolution of $A$ is an exact sequence of $G$-modules

$$
0 \rightarrow N \rightarrow Q \rightarrow A \rightarrow 0
$$

where $Q$ is a permutation module and $N$ is coflasque.

It turns out that there is a very direct relation between the group $\mathrm{H}^{1}(k, \operatorname{Pic} \bar{X})$ and flasque resolutions of the $G$-module $\hat{T}$, as the following result shows. 
Theorem 3.2 (Colliot-Thélène \& Sansuc). Let $T$ be a torus defined over a number field $k$ and split by a finite Galois extension $F / k$ with $G=\operatorname{Gal}(F / k)$. Suppose that

$$
0 \rightarrow \hat{T} \rightarrow P \rightarrow M \rightarrow 0
$$

is a flasque resolution of the $G$-module $\hat{T}$ and let $X / k$ be a smooth projective model of $T$. Then, we have

$$
\mathrm{H}^{1}(k, \operatorname{Pic} \bar{X})=\mathrm{H}^{1}\left(G, \operatorname{Pic} X_{F}\right)=\mathrm{H}^{1}(G, M) .
$$

Proof. See Lemme 5 and Proposition 6 of [4].

We proceed by presenting a very useful description of the group $\mathrm{H}^{1}(G, M)$ in the conclusion of the previous theorem.

Proposition 3.3. $\mathrm{H}^{1}(G, M)=\amalg_{\omega}^{2}(G, \hat{T})$.

Proof. See Proposition 9.5(ii) of [5].

Using this characterization, we can now prove Theorem 1.1 for $n \neq 6$ (the case $n=6$ will be treated separately in the next section).

Proof of Theorem 1.1 for $n \neq 6$. Set $G=\operatorname{Gal}(F / k) \cong A_{n}$ and $H=\operatorname{Gal}(F / K)$. Observe that such a group $H$ is necessarily isomorphic to $A_{n-1}$, since it has index $n$ in $A_{n}$. We have two cases:

Case $\boldsymbol{n} \geq \mathbf{8}$ : By Theorems 3.1 and 3.2 and Proposition 3.3, it is enough to establish that the group $\amalg_{\omega}^{2}(G, \hat{T})$ is trivial, where $T=R_{K / k}^{1} \mathbb{G}_{m}$ is the norm one torus associated to the extension $K / k$. Moreover, it is a well-known fact that $\hat{T}=J_{G / H}$ as $G$-modules, so it is sufficient to prove that $\amalg_{\omega}^{2}\left(G, J_{G / H}\right)=0$. But this was shown in Proposition 2.8 of Section 2, so the result follows.

Cases $n=5$ and $n=7$ : Since $n$ is a prime number, these cases follow from a direct application of Proposition 9.1 of [5]. In this proposition, the authors show that there exists a $k$-torus $T_{1}$ such that the variety $T \times_{k} T_{1}$ is $k$-rational, where $T=R_{K / k}^{1} \mathbb{G}_{m}$ is the norm one torus associated to $K / k$. This result is in its turn equivalent to the fact that any flasque module $M$ in a flasque resolution of $\hat{T}$ is invertible (see Proposition 9.5(i) of [5]), which is a stronger property than being coflasque. Therefore, the group $\mathrm{H}^{1}(G, M)$ vanishes and so, by Theorem 3.2, the middle group of Voskresenskiı̀'s exact sequence in Theorem 3.1 is trivial. Hence, we conclude that $A(T)=0=\amalg(T)$, as desired.

\section{THE CASE $n=6$}

In this section, we finish the proof of Theorem 1.1 by using the computer algebra system GAP to establish the remaining case $n=6$. More precisely, we devise an algorithm that, given a finite group $G$ and a non-normal subgroup $H$ such that $\operatorname{Core}_{G}(H):=\bigcap_{g \in G} g^{-1} H g$ is trivial (for example, this is always the case 
if $G$ is simple), outputs the invariant $\mathrm{H}^{1}(G, M)$ of Theorem 3.2 for the norm one torus. We use two ingredients to achieve this: First, we construct a routine in GAP that computes the matrix representation of the action of $G$ on the Chevalley module $J_{G / H}$. Second, we make use of the GAP algorithms ${ }^{1}$ developed by Hoshi and Yamasaki in [13] to construct flasque resolutions. Before we present our method, we need a few preliminaries.

Definition 4.1 (Definition 1.26 of [13]). Let $G$ be a finite subgroup of $\operatorname{GL}(n, \mathbb{Z})$. The $G$-lattice $M_{G}$ is defined to be the $G$-lattice with a $\mathbb{Z}$-basis $\left\{u_{1}, \ldots, u_{n}\right\}$ and right action of $G$ given by $u_{i} . g=\sum_{j=1}^{n} a_{i, j} u_{j}$, where $g=\left[a_{i, j}\right]_{i, j=1}^{n} \in G$.

In [13] the authors study the rationality of low-dimensional algebraic tori via the properties of the corresponding group modules, for which they create multiple algorithms. In particular, given a finite subgroup $G$ of $\operatorname{GL}(n, \mathbb{Z})$, they design the functions $\mathrm{H} 1$ and FlabbyResolution (see Sections 5.0 and 5.1 of [13, respectively) computing the cohomology group $\mathrm{H}^{1}\left(G, M_{G}\right)$ and producing a flasque resolution of the $G$-module $M_{G}$, respectively. For instance, by invoking the command

gap> FlabbyResolution(G).actionF;

in GAP, one can access the matrix representation of the action of $G$ on a flasque module in a flasque resolution of $M_{G}$.

Let $G$ be a finite group and $H$ a non-normal subgroup of $G$ with trivial normal core $\operatorname{Core}_{G}(H)$. Set $d=|G / H|$ and fix a set of right-coset representatives $L=\left\{H g_{1}, \ldots, H g_{d}\right\}$ of $H$ in $G$. In this way, we have $\mathbb{Z}[G / H]=\sum_{i=1}^{d} H g_{i} \mathbb{Z}$ and $N_{G / H}=\sum_{i=1}^{d} H g_{i} \in \mathbb{Z}[G / H]$.

Our first goal is to establish an isomorphism between the $G$-module $J_{G / H}$ and the $R_{G}$-module $M_{R_{G}}$, where $R_{G} \leq \mathrm{GL}(d-1, \mathbb{Z})$ is a group (to be defined below) isomorphic to $G$. We accomplish this by using the representation of $G$ associated to its right action on $J_{G / H}$. More precisely, consider the $\mathbb{Z}$-basis

$$
B=\left\{H g_{1}+N_{G / H} \mathbb{Z}, \ldots, H g_{d-1}+N_{G / H} \mathbb{Z}\right\}
$$

of $J_{G / H}$. Since the submodule $N_{G / H} \mathbb{Z}$ is fixed by the action of any element of $G$, we will omit it when working with elements of $B$. Given $g \in G$, we build a matrix $R_{g} \in \mathrm{GL}(d-1, \mathbb{Z})$ as follows.

For any $H g_{i} \in B$, we have $\left(H g_{i}\right) \cdot g=H g_{\sigma(i)}$ for some $1 \leq \sigma(i) \leq d$. There are two cases:

1) If $\sigma(i)<d$, then the $k$-th entry of the $i$-th row of $R_{g}$ is set to be equal to 1 if $k=\sigma(i)$ and 0 otherwise.

2) If $\sigma(i)=d$, then the $k$-th entry of the $i$-th row of $R_{g}$ is set to be equal to -1 for every $k$.

\footnotetext{
${ }^{1}$ The code for these algorithms is available on the web page https://www.math.kyoto-u. ac.jp/ yamasaki/Algorithm/RatProbAlgTori/
} 
Let $R_{G}$ be the group $\left\langle R_{g} \mid g \in G\right\rangle \leq \mathrm{GL}(d-1, \mathbb{Z})$. It is easy to see that the map

$$
\begin{aligned}
\rho_{G}: G & \longrightarrow R_{G} \\
g & \longmapsto R_{g}
\end{aligned}
$$

is the representation of $G$ corresponding to its action on $J_{G / H}$. Clearly we have ker $\rho_{G}=\operatorname{Core}_{G}(H)$, which we are assuming is trivial. Hence, $\rho_{G}$ is faithful and thus it yields an isomorphism $G \cong R_{G}$. Moreover, identifying $R_{g} \in R_{G}$ with the corresponding element $g \in G$, it is straightforward to check that the map

$$
\begin{aligned}
\psi: & M_{R_{G}} \longrightarrow J_{G / H} \\
& \sum_{i=1}^{d-1} \lambda_{i} u_{i} \longmapsto \sum_{i=1}^{d-1} \lambda_{i} H g_{i}+N_{G / H} \mathbb{Z}
\end{aligned}
$$

defines an isomorphism of group modules.

With the tools introduced so far, we are now able to construct the function FlasqCoho $(\mathrm{G}, \mathrm{H})$ (presented in the Appendix) in GAP that computes the cohomology group $\mathrm{H}^{1}(G, M)$, where $M$ is a flasque module in a flasque resolution of the Chevalley module $J_{G / H}$. The necessary steps to assemble this function are the following.

Step 1) Fix a set gens of generators of $G$ and construct the matrix group $R_{G}=\left\langle R_{g}\right| g \in$ gens $\rangle$ using the following two functions

- row $(\mathrm{s}, \mathrm{d})$ (an auxiliary routine to action), returning the $i$-th row of the matrix $R_{g}$ as explained on page 9 ;

- action $(\mathrm{G}, \mathrm{H})$, constructing the matrices $R_{g}$ for $g \in$ gens and returning the group $R_{G}$.

The code for these two functions is also provided in the Appendix. The group $R_{G}=\operatorname{action}(\mathrm{G}, \mathrm{H})$ is then a subgroup of $\mathrm{GL}(d-1, \mathbb{Z})$ isomorphic to $G$ such that $M_{R_{G}} \cong J_{G / H}$.

Step 2) Create a flasque resolution of the $R_{G}$-module $M_{R_{G}}$ and access its flasque module $M^{\prime}$ using the commands

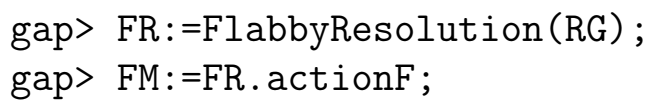

The object FM is the matrix representation group of the action of $R_{G}$ on $M^{\prime}$. Note that, by the inflation-restriction exact sequence, we have $\mathrm{H}^{1}\left(R_{G}, M^{\prime}\right) \cong$ $\mathrm{H}^{1}\left(\mathrm{FM}, M_{\mathrm{FM}}\right)$.

Step 3) Obtain the group $\mathrm{H}^{1}\left(\mathrm{FM}, M_{\mathrm{FM}}\right) \cong \mathrm{H}^{1}(G, M)$ using the function $\mathrm{H} 1$. gap> H1 (FM) ;

The result of this line is the final output of the algorithm. 
Using this computational method, we can now establish the remaining case of Theorem 1.1.

Proof of the case $n=6$ in Theorem 1.1. Set $G=\operatorname{Gal}(F / k) \cong A_{6}$ and $H=$ $\operatorname{Gal}(F / K)$. By Theorems 3.1 and 3.2, it is enough to prove that the cohomology group $\mathrm{H}^{1}(G, M)$ is trivial, where $M$ is a flasque module in a flasque resolution of the $G$-module $\hat{T}=J_{G / H}$ and $T=R_{K / k}^{1} \mathbb{G}_{m}$ is the norm one torus associated to the extension $K / k$.

As in the case $n \neq 6$, we have $H \cong A_{5}$. Notice that, up to conjugation, there are exactly two distinct subgroups of $A_{6}$ isomorphic to $A_{5}$, namely

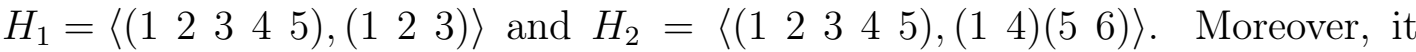
suffices to check the vanishing of $\mathrm{H}^{1}(G, M)$ for one subgroup $H$ in each conjugacy class (this follows from the fact that two subgroups $H_{1}$ and $H_{2}$ are conjugate if and only if the two $G$-sets $G / H_{1}$ and $G / H_{2}$ are isomorphic). Using the above algorithm, we obtained $\mathrm{H}^{1}(G, M)=0$ in both cases, as desired.

Remark 4.2. The computation used for the case $n=6$ in the previous proof can be reproduced for other small values of $n$. We have checked that for $n \leq 11$ the algorithm confirms our results, giving the trivial group for $n \neq 4$ and producing the counterexample $\mathrm{H}^{1}\left(A_{4}, M\right)=\mathbb{Z} / 2$ for $n=4$, as computed by Kunyavskiu in [15.

The authors of [13] also pay special attention to the case $n=5$ (see Example 8.1 of [13]). In this case, they establish that the torus $T=R_{K / k}^{1} \mathbb{G}_{m}$ is stably $k$-rational (see Corollary 1.11 of [13]), i.e. that there exists $n \in \mathbb{N}$ such that $T \times{ }_{k} \mathbb{G}_{m}^{n}$ is $k$-rational. In the language of group modules, this is equivalent to any flasque module $M$ in a flasque resolution of $\hat{T}$ being a permutation module, which is a stronger property than being coflasque.

The computational method developed in this section might be of independent interest, as it can often be used to compute the birational invariant $\mathrm{H}^{1}(G, M)$ for low-degree field extensions and, in this way, deduce consequences about the groups $A(T)$ and $\mathfrak{K}(K / k)$. 


\section{APPENDIX}

Remark 4.3. The code for all the functions below can also be found at https: //sites.google.com/view/andre-macedo/code. Additionally, in order to successfully run the function FlasqCoho, the user will need the GAP programs for the functions ConjugacyClassesSubgroups2, H1 and FlabbyResolution (see Sections 4.1, 5.0 and 5.1 of [13], respectively).

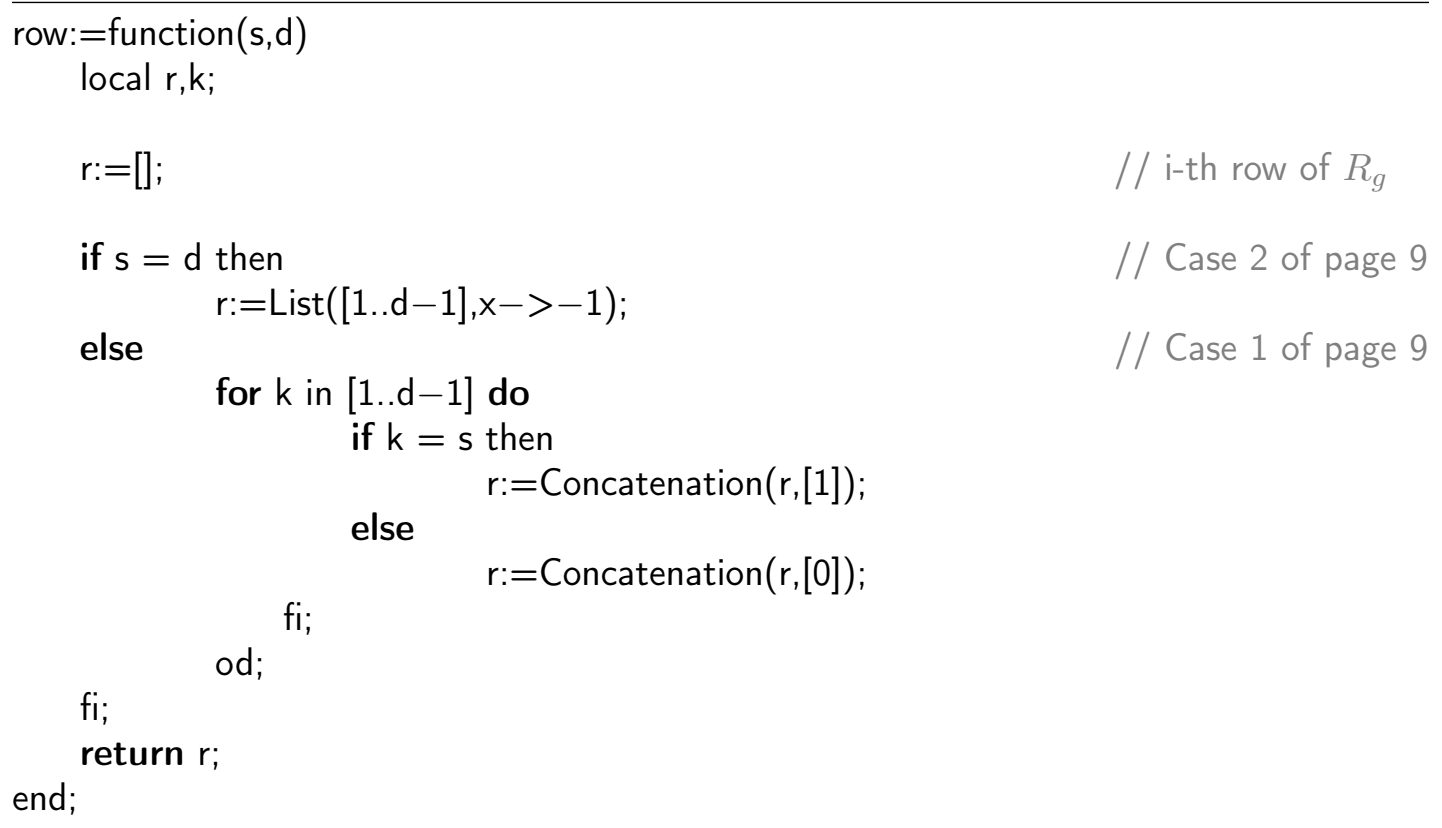

action: $=$ function $(\mathrm{G}, \mathrm{H})$

local d,gens, RT,L,S,j, Rg,i,s;

$\mathrm{d}:=\operatorname{Order}(\mathrm{G}) / \operatorname{Order}(\mathrm{H})$;

gens:=GeneratorsOfGroup $(\mathrm{G})$;

RT:=RightTransversal $(\mathrm{G}, \mathrm{H})$;

$\mathrm{L}:=$ List $(\mathrm{RT}, \mathrm{i}->$ CanonicalRightCosetElement $(\mathrm{H}, \mathrm{i}))$;

// List of right-coset representatives of $H$ in $G$

$\mathrm{S}:=[]$

// List of matrices $R_{g}$ for $g \in$ gens

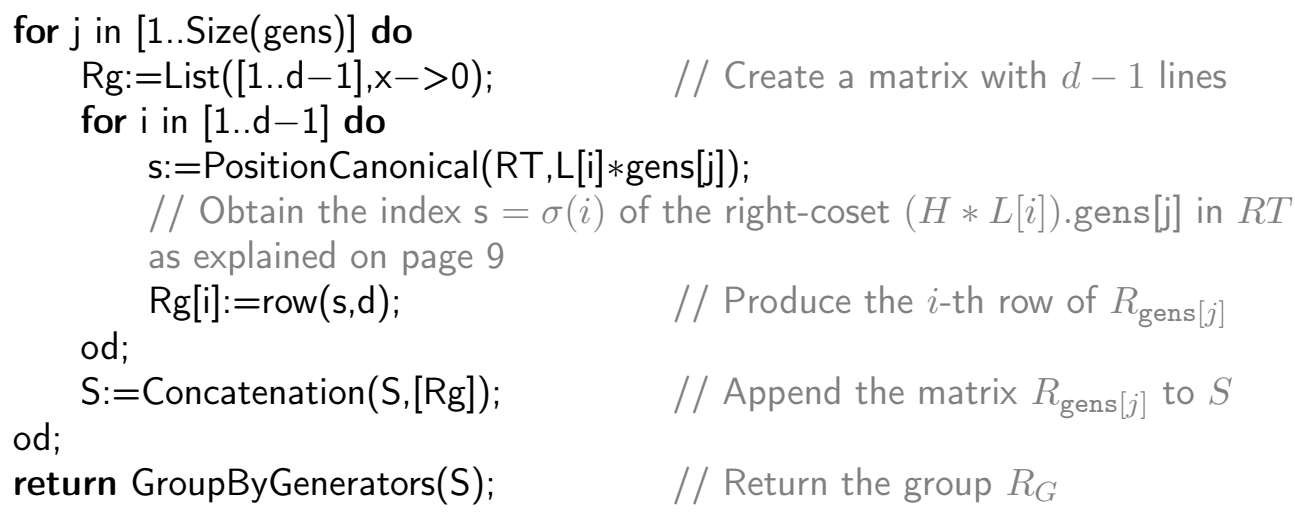




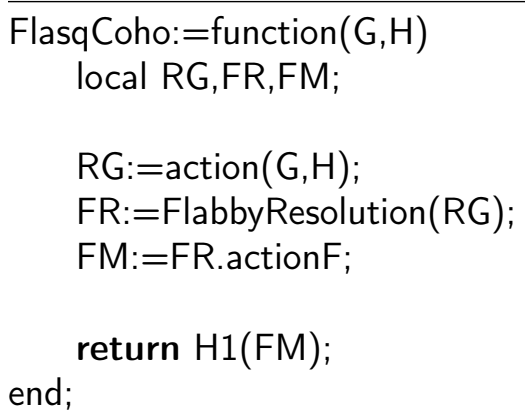

// Matrix group $R_{G}$

// Flasque resolution of $R_{G}$

// Flasque module in FR

// Return the cohomology group $\mathrm{H}^{1}(G, M)$

\section{REFERENCES}

[1] H.-J. Bartels, Zur Arithmetik von Konjugationsklassen in algebraischen Groupen, J. Algebra 70 (1981), 179-199.

[2] H.-J. Bartels, Zur Arithmetik von Diedergruppenerweiterungen, Math. Ann. 256 (1981), 465-473.

[3] K. Brown, Cohomology of groups, Graduate Texts in Mathematics 87, Springer-Verlag, 1982.

[4] J.-L. Colliot-Thélène, J.-J. Sansuc, La R-équivalence sur les tores. Ann. Sc. E.N.S. 10 (1977), 175-229.

[5] J.-L. Colliot-Thélène, J.-J. Sansuc, Principal homogeneous spaces under flasque tori: Applications, J. Algebra 106 (1987), 148-205.

[6] Y. A. Drakokhrust, V. P. Platonov, The Hasse norm principle for algebraic number fields, Math. USSR-Izv. 29 (1987), 299-322.

[7] C. Frei, D. Loughran, R. Newton, The Hasse norm principle for abelian extensions, Amer. J. Math, to appear.

[8] The GAP Group, GAP - Groups, Algorithms, and Programming, Version 4.8.10 (2018), available at https://www.gap-system.org

[9] F. Gerth, The Hasse norm principle for abelian extensions of number fields, Bulletin of the AMS, Vol. 83 (1977), 264-266.

[10] K. W. Gruenberg, Cohomological Topics in Group Theory, Lecture Notes in Mathematics 143, Springer-Verlag, 1970.

[11] P. N. Hoffman, J. F. Humphreys, Projective representations of the symmetric groups, Oxford Mathematical Monographs, Clarendon Press, Oxford, 1992.

[12] M. Horie, The Hasse norm principle for elementary abelian extensions, Proc. Amer. Math. Soc. 118(1) (1993), 47-56.

[13] A. Hoshi, A. Yamasaki, Rationality problem for algebraic tori, Memoirs of the American Mathematical Society 248 (2017), no. 1176.

[14] G. Karpilovsky, The Schur Multiplier, Clarendon Press, Oxford, 1987.

[15] B. Kunyavskiı̌, Arithmetic properties of three-dimensional algebraic tori, Zap. Nauch. Sem. LOMI Akad. Nauk SSSR 16 (1982), 102-107.

[16] J. S. Milne, Class Field Theory, Version 4.02 (2013), available at http://www.jmilne. org/math/CourseNotes/CFT .pdf.

[17] V. Platonov, A. Rapinchuk, Algebraic groups and number theory, Pure and Applied Mathematics, 139, Academic Press, Inc., Boston, MA, 1994.

[18] J. Schur, Über die Darstellung der symmetrischen und der alternierenden Gruppe durch gebrochene lineare Substitutionen, J. reine angew. Math. 139 (1911), 155-250.

[19] V. Voskresenskiı̌, Birational properties of linear algebraic groups, Izv. Akad. Nauk SSSR Ser. Mat. 34 (1970) 3-19. English translation: Math. USSR-Izv. Vol. 4 (1970), 1-17.

[20] V. Voskresenskiı̌, B. Kunyavskiı̌, Maximal tori in semisimple algebraic groups, Manuscript deposited at VINITI 15.03.84, no. 1269-84, 28pp. (in Russian).

[21] R. A. Wilson, The finite simple groups, Graduate Texts in Mathematics 251, SpringerVerlag, 2009. 
André Macedo, Department of Mathematics and Statistics, University of Reading, Whiteknights, PO Box 220, Reading RG6 6AX, UK

E-mail address: c.a.v.macedo@pgr.reading.ac.uk

$U R L:$ https://sites.google.com/view/andre-macedo 